\title{
Simulation model based approach for long exposure atmospheric point spread function reconstruction for laser guide star multiconjugate adaptive optics
}

\author{
Luc Gilles, ${ }^{1, \star}$ Carlos Correia, ${ }^{2}$ Jean-Pierre Véran, ${ }^{2}$ Lianqi Wang,,${ }^{1}$ and Brent Ellerbroek ${ }^{1}$ \\ ${ }^{1}$ Thirty Meter Telescope Observatory Corporation, 1200 East California Boulevard, \\ MC 102-8, Pasadena, California 91125, USA \\ ${ }^{2}$ National Research Council Canada, Herzberg Institute of Astrophysics, 5171 West Saanich Road, \\ Victoria, British Columbia V9E 2E7, Canada \\ *Corresponding author: Igilles@ caltech.edu
}

Received 6 July 2012; revised 21 September 2012; accepted 24 September 2012; posted 24 September 2012 (Doc. ID 172096); published 22 October 2012

\begin{abstract}
This paper discusses an innovative simulation model based approach for long exposure atmospheric point spread function (PSF) reconstruction in the context of laser guide star (LGS) multiconjugate adaptive optics (MCAO). The approach is inspired from the classical scheme developed by Véran et al. [J. Opt. Soc. Am. A 14, 3057 (1997)] and Flicker et al. [Astron. Astrophys. 400, 1199 (2003)] and reconstructs the long exposure optical transfer function (OTF), i.e., the Fourier transformed PSF, as a product of separate long-exposure tip/tilt removed and tip/tilt OTFs, each estimated by postprocessing system and simulation telemetry data. Sample enclosed energy results assessing reconstruction accuracy are presented for the Thirty Meter Telescope LGS MCAO system currently under design and show that percent level absolute and differential photometry over a 30 arcsec diameter field of view are achievable provided the simulation model faithfully represents the real system. (C) 2012 Optical Society of America

OCIS codes: $\quad 010.1080,010.1330$.
\end{abstract}

\section{Introduction}

Point spread function (PSF) knowledge is critical for any existing or proposed adaptive optics (AO) astronomical science program aiming at obtaining high angular resolution information. Examples of such programs include photometry and astrometry in crowded and sparse stellar fields, detection and characterization of exoplanets, determination of precision orbits at the Galactic Center to test general relativity and black hole growth models, dynamics of early galaxies, and gravitational lensing [1-5]. In order to enable these science programs, $\overline{\mathrm{AO}}$ systems on existing and future extremely large telescopes are

1559-128X/12/317443-16\$15.00/0

(C) 2012 Optical Society of America required to meet tight photometry and astrometry budgets. For instance, the laser guide star (LGS) multiconjugate adaptive optics (MCAO) system, under design for the Thirty Meter Telescope (TMT), is required to provide $2 \%$ differential photometry (relative brightness between two point sources) over a 30 arcsec diameter field of view $(\mathrm{FoV})$ for a $10 \mathrm{~min}$ integration at a wavelength of $1 \mu \mathrm{m}$, and 50 microarcsec root-meansquare (RMS) time dependent differential astrometry (relative separation between two point sources) over the same FoV for a $100 \mathrm{~s}$ integration in $\mathrm{H}$ band [6,7].

The mathematical quantity that is actually determined in PSF reconstruction (PSFR) is the secondorder statistics of the residual phase, namely the structure function (SF), i.e., the variance of the differential phase between two points in the telescope 
aperture. The optical transfer function (OTF), i.e., the normalized Fourier transform of the PSF, is then computed from the SF via the long exposure formula (long exposure means that the residual speckle noise is assumed to have completely averaged out):

$$
\widehat{\mathrm{OTF}}(u)=\Phi^{-1} \int \mathrm{d}^{2} x A(x) A(x+\lambda u) e^{-\hat{D}(x, x+\lambda u) / 2},
$$

where hats denote reconstructed quantities, $\Phi=$ $\int \mathrm{d}^{2} x A^{2}(x)$ is the PSF flux, $u=\left(u_{x}, u_{y}\right)$ is the focal plane angular frequency coordinate, $A(x)$ is the real-valued telescope pupil function, and $D\left(x, x^{\prime}\right)$ denotes the residual phase SF between two apertureplane points with coordinates $x$ and $x^{\prime}$ (units of $\operatorname{rad}^{2}$ ), i.e.,

$$
D\left(x, x^{\prime}\right)=\left\langle\left[\phi(x)-\phi\left(x^{\prime}\right)\right]^{2}\right\rangle,
$$

where angle brackets denote temporal averaging and $\phi(x)$ the residual aperture-plane phase at coordinate $x$ (assumed to have zero mean). For a telescope with a circular aperture of diameter $D_{0}$, the OTF has a circular support of radius equal to $D_{0} / \lambda$ (telescope cutoff frequency). The diffraction limited OTF is simply expressed as in Eq. (1) with $D\left(x, x^{\prime}\right)=0$, i.e.,

$$
\mathrm{OTF}_{D L}(u)=\Phi^{-1} \int \mathrm{d}^{2} x A(x) A(x+\lambda u) .
$$

The reconstructed PSF is obtained by the inverse Fourier transform of Eq. (1):

$$
\widehat{\operatorname{PSF}}(\theta)=\Phi F^{-1}\{\widehat{\mathrm{OTF}}(u)\}(\theta),
$$

where $\theta=\left(\theta_{x}, \theta_{y}\right)$ is the focal plan angular coordinate (conjugate variable to $u$ ), and $F^{-1}$ denotes the inverse Fourier transform operator. The estimated Strehl ratio (SR) and enclosed energy (EE) are given by the following expressions:

$$
\begin{aligned}
\widehat{\mathrm{SR}} & =\frac{\widehat{\operatorname{PSF}}(0)}{\operatorname{PSF}_{D L}(0)}=\frac{\int \mathrm{d}^{2} u \widehat{\mathrm{OTF}}(u)}{\int \mathrm{d}^{2} u \mathrm{OTF}_{D L}}, \widehat{\mathrm{EE}}(\Omega) \\
& =\Phi^{-1} \int_{-\Omega / 2}^{\Omega / 2} \mathrm{~d} \theta_{x} \int_{-\Omega / 2}^{\Omega / 2} \mathrm{~d} \theta_{y} \widehat{\operatorname{PSF}}(\theta) \\
& =\Omega^{2} \int \mathrm{d}^{2} u \widehat{\mathrm{OTF}}(u) \operatorname{sinc}\left(u_{x} \Omega\right) \operatorname{sinc}\left(u_{y} \Omega\right),
\end{aligned}
$$

where $\Omega$ denotes the angular width of the square integration domain. The reconstructed atmospheric AO OTF [Eq. (1)] is real-valued, i.e., it assumes a zero temporal mean phase, an assumption that leads to a symmetric PSF, i.e., $\widehat{\operatorname{PSF}}(\theta)=\widehat{\operatorname{PSF}}(-\theta)$. Modeling of static telescope and optomechanical phase aberrations (common and noncommon path) is possible, however, reconstruction of such biases (which produce a nonsymmetrical PSF) requires the use of phase diversity techniques [묘, $\underline{9}$ (possibly tomographic techniques for aberrations at nonzero conjugation altitudes), and is not studied in this paper.
Véran pioneered AO PSFR in 1997 by developing a practical method to reconstruct the long exposure OTF of a bright natural guide star (NGS) target from the wavefront sensor (WFS) measurement covariance matrix accumulated during the exposure [10]. There is no anisoplanatism for such an observation since the NGS is the science target. Fusco et al. in 2000 [11], and later Britton et al. in 2005 [12], extended Véran's technique to capture a key missing component: angular anisoplanatism, providing the astronomical AO community a complete PSFR tool for classical NGS single-conjugate AO (SCAO) observations. SR errors of $4 \%$ in $\mathrm{K}$-band are reported on the Palomar AO system (order $16 \times 16$ ) at the $5 \mathrm{~m}$ Hale Telescope for observations of a bright star separated by 21 arcsec from its companient star (28\% measured companient SR versus $27 \%$ predicted SR), and 100 microarcsec differential astrometry for a 2 min exposure using 50 reference stars. Measurements of the turbulence profile provided the necessary input for the angular anisoplanatism filter computation in these experiments. Recently, Jolissaint reported 5\% K-band SR error at the $10 \mathrm{~m} \mathrm{Keck}$ Telescope for bright NGS AO observations [8] and stressed two critical steps to achieve this level of performance: estimation of the static/quasi-static optical aberrations (common and non-common path), and knowledge of the atmospheric turbulence profile. Flicker, in 2008, pushed the theory further by developing an analytical PSFR algorithm for LGS SCAO, estimating the long exposure OTF as a product of separate long exposure tip/tilt removed (TTR) and tip/tilt (TT) OTFs, the former including the effect of focal anisoplanatism (FA) [13] (a block diagram is given in Fig. 2 of Section 2).

To the best of our knowledge, PSFR for multi-LGS tomography AO systems has been discussed so far only for the ground-layer AO (GLAO) facility system under design for the large binocular telescope [14]. For LGS MCAO, multiple low-order NGS WFSs are required to correct low-order tomographic nullmodes invisible to the high-order LGS WFSs but producing absolute and differential magnification on the science focal plane [15], commonly referred to as tip/ tilt anisoplanatism (TTA). More importantly, the effects of angular and focal anisoplanatism are very different from what they are for LGS GLAO and SCAO: the PSF is much more uniform across an extended FoV (characterized by the generalized isoplanatic angle [16]) and the TTR SR of a point source at infinity (science target) is actually larger than that of a point source in the same direction but at finiterange (the LGS of the multi-LGS constellation pointing to the science target), since the deformable mirror (DM) fitting step is optimized for the science target at infinity, not for the finite range LGSs. The LGS OTF thus has to be boosted by some amount greater than unity to yield the science TTR OTF, meaning that the latter cannot be expressed as a product of individual OTF error terms, and crosscoupling terms need to be taken into account. All this 

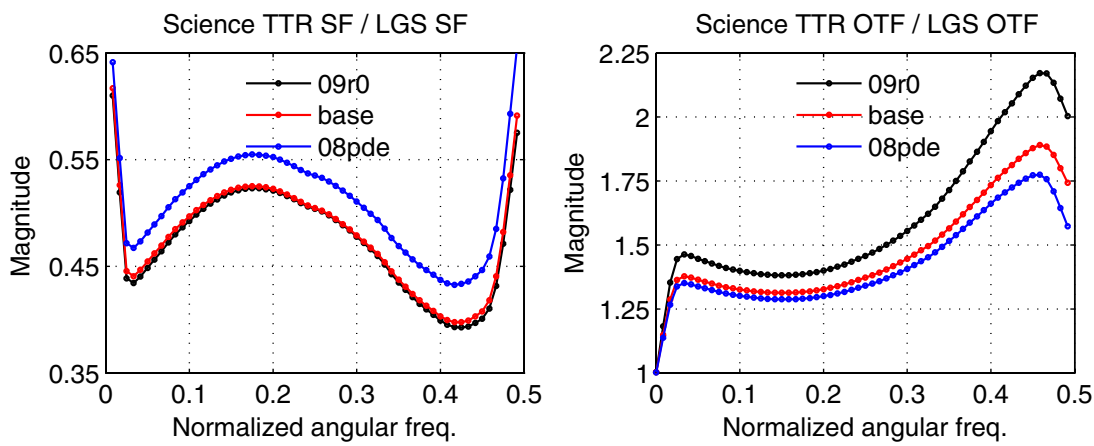

SF Ratio

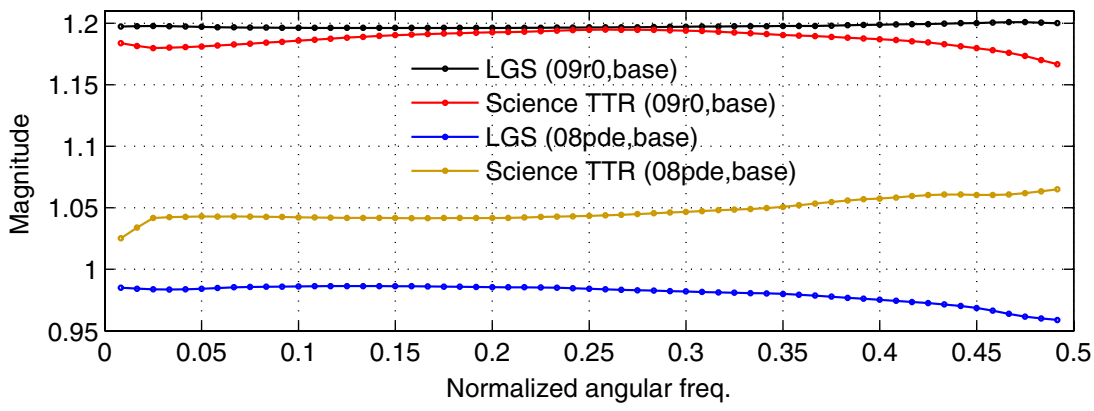

Fig. 1. (Color online) Upper left panel shows science TTR to LGS SF for the TMT LGS MCAO system for three observing conditions: baseline (base), $10 \%$ reduced Fried parameter (09r0), and 20\% reduced LGS WFS signal level (08pde). Note that the magnitude of these SF ratios is smaller than unity, the SF ratio is insensitive to seeing variations, but sensitive to LGS WFS signal level variations. Upper right panel shows science TTR to LGS OTF ratios computed from the SFs illustrated on the left panel. Bottom panel shows LGS and science TTR SF ratios to illustrate how SFs vary with seeing and LGS WFS signal level.

makes an analytic derivation very difficult, if possible at all. This behavior, as well as other differences between the LGS and the science TTR SFs and OTFs, are illustrated in Fig. 1. Important features to note are that the science TTR to LGS SF ratio [i.e., the extrapolation filter described in Eq. (15)] is smaller than unity (i.e., the LGS SR is smaller than the science TTR SR), insensitive the seeing variations (i.e., the filter is robust against seeing modeling errors), but sensitive to LGS WFS signal level (opposite behaviors of the science TTR and LGS SF make the filter sensitive to this type of modeling error). In contrast to SF ratios, OTF ratios are sensitive to seeing variations.

Inspired from Véran et al. [10] and Flicker et al. [17], the approach described in this paper reconstructs the LGS MCAO long exposure OTF as a product of separate TTR and TT components, each estimated from a combination of AO system telemetry data as well as simulation model data (a block

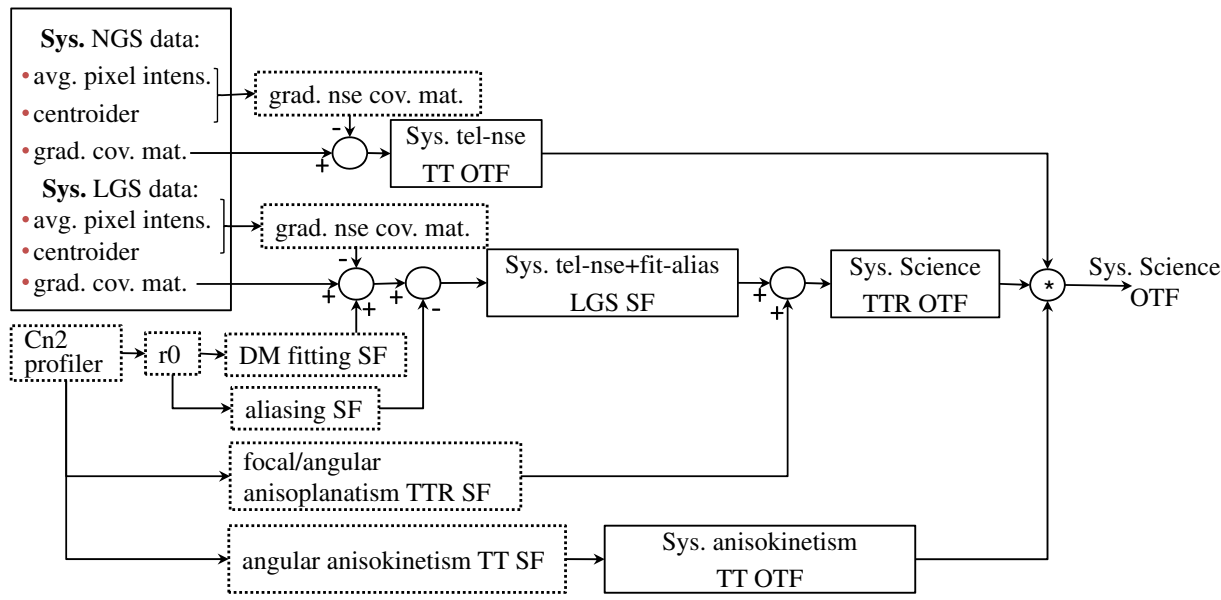

Fig. 2. (Color online) Block diagram of the classical LGS SCAO PSFR scheme, illustrating the main steps involved in the algorithm. The acronym "Sys." has been prefixed to quantities related to or extracted from the AO system telemetry data. This notation is useful to distinguish quantities derived from simulation models. Static and quasi-static slowly varying telescope and AO phase errors, e.g., optomechanical errors, residual sodium layer tracking errors, etc., are omitted at this stage of the study. 


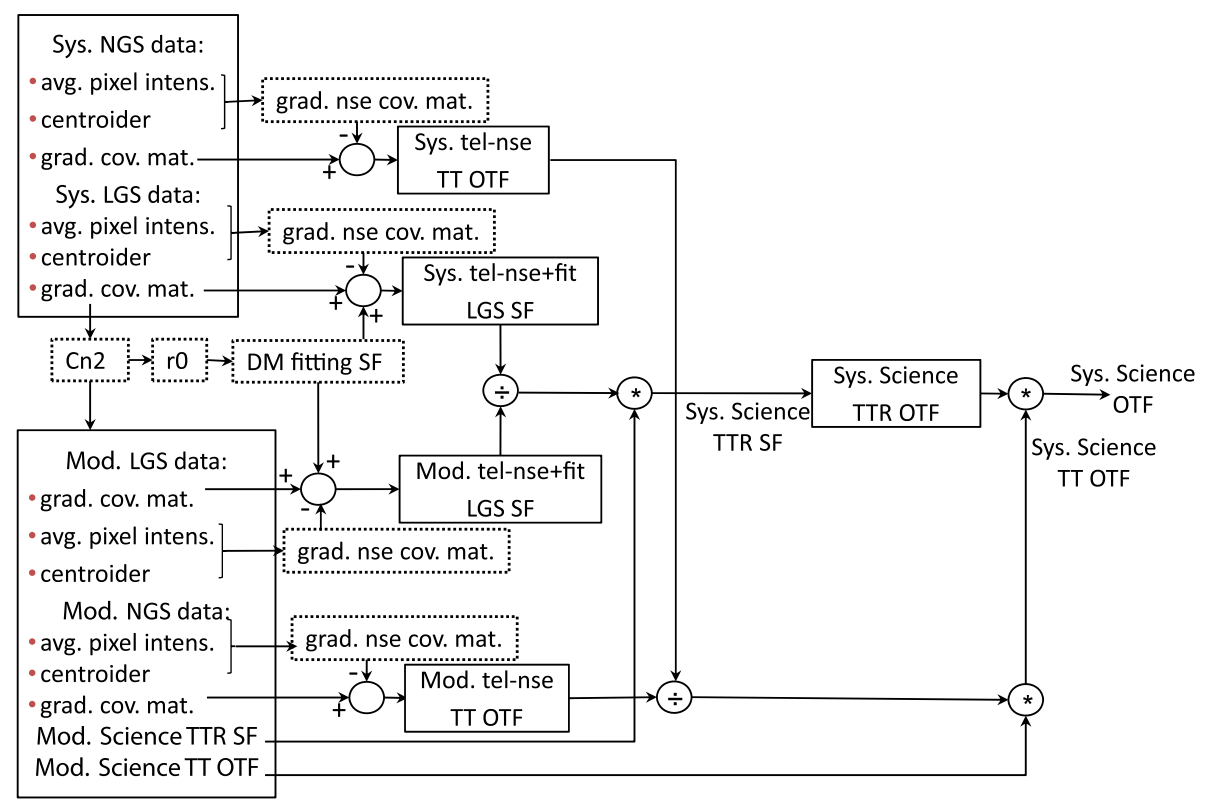

Fig. 3. (Color online) Block diagram of the proposed LGS MCAO PSFR scheme, illustrating the main steps involved in the algorithm. The acronyms "Sys." and "Mod." have been prefixed to quantities related to, or extracted from, either the real AO system or the simulation model telemetry data. Static and quasi-static slowly varying telescope and AO phase errors are omitted at this stage of the study.

diagram is given in Fig. 3 of Section 2 ). Namely, an LGS SF is reconstructed from the measurement covariance matrix of the LGS WFS pointing to the center of the science FoV (we will call this direction "on-axis"). It is then extrapolated to the science target (direction and infinite range) using a precomputed filter obtained from a high-fidelity simulation model of the full system. A science TTR OTF is then computed from the science TTR SF, and is finally multiplied by a science TT OTF estimated from the global measurement covariance matrix of multiple low-order TT NGS WFSs typically patrolling a larger field than the science FoV.

The paper is organized as follows. Section 2 provides a review of the classical LGS SCAO PSFR scheme and draws upon it to introduce our proposed simulation model based approach for LGS MCAO. Performance evaluation is presented in Section $\underline{3}$, and conclusions are given in Section $\underline{4}$.

\section{Simulation Model Based Approach to PSFR for LGS MCAO}

\section{A. Review of Classical Scheme for LGS SCAO}

The integrand in Eq. (1) is typically discretized on a nodal aperture-plane grid whose sampling $\Delta x$ is equal to the WFS subaperture size, $d_{\mathrm{SA}}$, and $u$ is discretized on a square grid of width equal to $2 D_{0} / \lambda$ with sampling $\Delta u=\Delta x / \lambda$. The number of grid points in a single dimension is thus equal to twice the WFS order, i.e., $n=2 D_{0} / \Delta x$. Equations (1) and (4) are thus arrays of size $n \times n$. Note that since the $\mathrm{S} \overline{\mathrm{F}} \hat{D}\left(x, x^{\prime}\right)$ in Eq. (1) is a four-dimensional (4D) quantity, its storage scales as the square of that of the OTF [which is only a two-dimensional (2D) quantity]. Namely, denoting by $N \sim n^{2}(\pi / 16) \sim n^{2} / 5$ the total number of reconstructed aperture-plane grid points inside the telescope aperture $\hat{D}$ can be stored in the form of a symmetric matrix of size $N \times N$. The correlation type integral, Eq. (1), is then computed for all spatial shifts $\lambda\left(u_{x}, u_{y}\right)$ by fetching the appropriate SF matrix elements. A Matlab routine is available from the authors to the interested readers wishing to implement the technique. As pointed by Flicker et al. [13], although it might have seemed computationally challenging at one time, Eq. (1) can nowadays easily be computed for extremely large telescope apertures on a modern laptop computer, and it is unnecessary to resort to the aperture-averaging approximation, i.e., approximating the $4 \mathrm{D} \mathrm{SF}$ by a $2 \mathrm{D} \mathrm{SF}$ as suggested by Véran et al. in [10]. From discrete Fourier transform properties, the reconstructed PSF, Eq. (4) is Nyquist sampled, i.e., its sampling is $\Delta \theta=\bar{\lambda} /\left(2 D_{0}\right)$, and it spans a total angular width equal to $1 /(\Delta u)=\lambda / \Delta x$

Flicker, in 2008, developed an analytical PSFR algorithm for LGS SCAO, estimating the science SF, $D\left(x, x^{\prime}\right)$ in Eq. (1) as the sum of separate TTR and TT SFs, both computed for a point source at infinite range in the science direction [13]:

$$
\hat{D}\left(x, x^{\prime}\right)=\hat{D}_{\mathrm{TTR}}\left(x, x^{\prime}\right)+\hat{D}_{\mathrm{TT}}\left(x-x^{\prime}\right),
$$

where $\hat{D}_{\text {TTR }}$ denotes the TTR SF, which, as described in Fig. 2, includes focal and angular anisoplanatism, and $D_{\mathrm{TT}}$ is the TT SF, which is shift-invariant, i.e., dependent only upon the $2 \mathrm{D}$ relative separation between two aperture-plane points, expressed as follows:

$$
\hat{D}_{\mathrm{TT}}(\lambda u)=16\left(\lambda / D_{0}\right)^{2} u^{T} \hat{C}_{\mathrm{TT}} u,
$$


where $\hat{C}_{\mathrm{TT}}$ denotes the reconstructed modal Zernike TT phase covariance matrix $\left(2 \times 2\right.$, in units of $\left.\operatorname{rad}^{2}\right)$. Substituting Eq. (ㅁ) into Eq. ()ㅜ) yields:

$$
\widehat{\mathrm{OTF}}(u)=\widehat{\mathrm{OTF}}_{\mathrm{TT}}(u) \widehat{\mathrm{OTF}}_{\mathrm{TTR}}(u),
$$

where $\widehat{\mathrm{OTF}}_{\mathrm{TTR}}$ is expressed as in Eq. (1) in terms of $\hat{D}_{\text {TTR }}$, and $\widehat{\mathrm{OTF}}_{\mathrm{TT}}$ is the TT OTF, expressed as:

$$
\widehat{\mathrm{OTF}}_{\mathrm{TT}}(u)=e^{-\hat{D}_{\mathrm{TT}}(\lambda u) / 2} \text {. }
$$

Equation (138) of the final report [13] provides the expression of the FA SF projected onto the DM influence functions (controlled modes), and should be used to compute the FA component of $\hat{D}_{\text {TTR }}\left(x, x^{\prime}\right)$ for LGS SCAO systems (LGS SCAO observations with e.g., the Keck, Gemini, Subaru, Lick, and Palomar telescopes). A block diagram of Flicker's approach, illustrating the main steps of his algorithm, is provided in Fig. 2.

The necessary telemetry data consists of (i) the accumulated measurement covariance matrix, (ii) the subaperture average pixel intensities, and (iii) the average centroider gain, for both the LGS and TT NGS WFSs. The LGS SF is reconstructed from the LGS WFS measurement covariance matrix. This step requires to "denoise" and "dealias" the measurement covariance matrix, as well as to add in the unseen DM fitting error. Denoising is performed by postprocessing the subaperture average pixel intensities and centroider gain, whereas "dealiasing" and "adding in fitting" is performed from a simulation model capturing the main WFS detector characteristics as well as the type of DM influence functions, and requires good knowledge of the Fried parameter, which can be obtained either from a dedicated turbulence profiler near the telescope, or from a DM seeing estimation technique recently validated on the sky at the Keck telescope [8]. Mathematically, these steps can be represented in the following form:

$\hat{C}_{\mathrm{LGS}}=R_{\mathrm{LGS}}\left(C_{g \mathrm{LGS}}-\hat{C}_{g \mathrm{LGSnse}}\right)\left(R_{\mathrm{LGS}}\right)^{T}-\hat{C}_{\text {alias }}+\hat{C}_{\text {fit }}$,

where $C_{g \mathrm{LGS}}$ denotes the LGS WFS measurement covariance matrix (subscript $g$ stands for gradient, we assume that the system uses a Shack-Hartmann WFS providing approximately twice as many measurements as reconstructed phase points in the Fried geometry), $\hat{C}_{g \text { LGSnse }}$ denotes the estimated measurement noise covariance matrix, obtained by postprocessing the subaperture average pixel intensities and centroider gain (see for instance [18] for formulas for a matched filter centroiding algorithm), $R_{\text {LGS }}$ is the gradient-to-phase reconstruction matrix, $\hat{C}_{\text {alias }}$ is the estimated $N \times N$ covariance matrix of the spatial aliasing error, and $\hat{C}_{\text {fit }}$ the estimated covariance matrix of the unseen DM fitting error. Both $\hat{C}_{\text {alias }}$ and $\hat{C}_{\text {fit }}$ are retrieved either from analytical formulas
[19], or a dedicated offline AO error budget simulation tool directly accumulating these type of phase error covariance matrices at coarse resolution $\left(\Delta x=d_{\mathrm{SA}}\right)$. The negative sign on the measurement noise covariance matrix in Eq. (10) has to be understood from the fact that measurement noise has to be removed, whereas that on the aliasing covariance matrix has been suggested by Correia for AO systems implementing minimum variance reconstruction matrices (whose regularization strongly attenuate aliasing) [20]. Note that the noise removal step, $C_{g \text { LGS }}-\hat{C}_{g \text { LGSnse }}$, may not always result in a positive semidefinite covariance matrix (eigenvalues greater or equal to zero), hence an eigenvalue decomposition is required to null eventual negative eigenvalues, and similarly for the aliasing removal step. The phase reconstruction matrix, $R_{\mathrm{LGS}}$, is typically assembled following a least-squares criteria, i.e.,

$$
R_{\mathrm{LGS}}=\left(\Gamma^{T} \Gamma\right)^{\dagger} \Gamma^{T},
$$

where $\Gamma$ denotes the phase-to-measurement influence matrix, and $\dagger$ denotes pseudoinverse. For a Shack-Hartmann WFS and a reconstruction grid in the Fried geometry (grid points located at subaperture vertices), wafflelike modes are poorly sensed and need typically to be filtered out via regularization. $\hat{C}_{\mathrm{LGS}}$ is then transformed into a SF matrix, which, by definition, is related to the latter as follows:

$$
\hat{D}_{\mathrm{LGS}}=\operatorname{diag}\left(\hat{C}_{\mathrm{LGS}}\right) 1^{T}+1 \operatorname{diag}\left(\hat{C}_{\mathrm{LGS}}\right)^{T}-2 \hat{C}_{\mathrm{LGS}},
$$

where 1 denotes the column vector of all $1 \mathrm{~s} . \hat{D}_{\mathrm{LGS}}$ is a $N \times N$ symmetric indefinite matrix (eigenvalues positive and negative) with zeros along the diagonal and positive values elsewhere. At this point, it remains to extrapolate the reconstructed LGS SF to the science target (direction and infinite range). We note that for a LGS SCAO system, FA leads to a residual TTR phase SF for a point source at infinite range that is larger in magnitude than that for a point source in the same direction but at finite range. The precomputed FA SF, Eq. (138) in [13], is thus summed to $\hat{D}_{\mathrm{LGS}}$ to yield the reconstructed science TTR SF, $\hat{D}_{\text {TTR }}$. Note that this step requires knowledge of the turbulence profile.

This terminates the processing of the high-order LGS data. We now turn to processing of the TT NGS data. A TT covariance matrix in the direction of the NGS is computed from the de-noised covariance matrix of the TT NGS WFS:

$$
\hat{C}_{\text {TTNGS }}=C_{g N G S}-\hat{C}_{g N G S n s e},
$$

where $C_{g N G S}$ denotes the NGS TT WFS $2 \times 2$ measurement covariance matrix, and $\hat{C}_{g \text { NGSnse }}$ denotes the estimated measurement noise covariance matrix, obtained by postprocessing the NGS TT WFS subaperture average pixel intensities and centroider 
gain. The TT covariance estimate Eq. (13) is finally extrapolated to the science direction by addition of a TT anisokinetism covariance term, computed from Eq. (110) of [13] by substitution of the Zernike TT modes for the weighting functions appearing inside the integral. The result is then transformed into the TT SF Eq. ()), and finally OTF Eq. (9).

\section{B. Extension to LGS MCAO}

The LGS MCAO approach described in this paper is inspired from the above classical LGS SCAO PSFR scheme. A block diagram is provided in Fig. 3.

An LGS SF, $\hat{D}_{\mathrm{LGS}}$, is reconstructed from the measurement covariance matrix of the LGS WFS pointing to the center of the science FoV following the steps described in Eqs. (10)-(12). Note that $R_{\text {LGS }}$ reconstructs the residual phase covariance matrix of only a single LGS WFS, and is therefore not a tomographic reconstruction matrix. In particular, it is unrelated to the tomographic reconstruction algorithm implemented in the real system.

Next, following Flicker et al. [17], a TT OTF for the science target (direction and infinite range) is estimated from the denoised global measurement covariance matrix of multiple low-order TT NGS WFSs patrolling typically a larger field than the science FoV, a procedure that generalizes the SCAO science TT OTF estimation described in Fig. $\underline{2}$ to the tomographic case. Mathematically

$$
\hat{C}_{\mathrm{TT}}=H_{\mathrm{TT}} R_{\mathrm{NGS}}\left(C_{g \mathrm{NGS}}-\hat{C}_{g \mathrm{NGSnse}}\right)\left(R_{\mathrm{NGS}}\right)^{T}\left(H_{\mathrm{TT}}\right)^{T},
$$

where $\hat{C}_{\text {TT }}$ denotes the estimated $2 \times 2$ TT Zernike covariance matrix for the science direction, $R_{\mathrm{NGS}}^{\mathrm{sys}}$ denotes the modal tomographic NGS phase reconstruction matrix, reconstructing typically five modes consisting of global TT and three quadratic modes defined on two layers from at least six measurements (provided by three TT NGS WFSs), and $H_{\mathrm{TT}}$ denotes the projection matrix onto local TT along the science direction of interest. Estimation of the NGS WFS measurement noise covariance matrix, $\hat{C}_{g N G S n s e}$ in Eq. (14), is performed by postprocessing the average detector pixel intensities and centroider gains from all NGS WFSs, also recorded as part of the telemetry data. Note that this denoising step may not always result in a positive semidefinite covariance matrix, hence an eigenvalue decomposition may be required to null eventual negative eigenvalues. The science TT OTF Eq. (9) is then computed by substituting Eq. (14) into Eq. (7). We find that the accuracy of this TT reconstruction strongly depends upon the NGS constellation (location and brightness of the NGSs), suggesting that either angular anisokinetism or high-order LGS-uncorrected aliasing is missing from Flicker's formula Eq. (14).

At this point, it remains to extrapolate the reconstructed LGS SF to the science target (direction and infinite range). We opted to base our extrapolation strategy on a high-fidelity simulation model of the full system. It is important to note that the simulation model requires knowledge of the atmospheric turbulence profile, in particular accurate knowledge of the Fried parameter. This profile can be estimated either offline by postprocessing the cross-covariance measurement matrix of a pair of LGSs from the multi-LGS constellation, or directly online. Such a turbulent profiling method has been demonstrated in simulations [21] and on the sky at the Gemini South telescope [22]. We will use superscript "mod" to indicate data extracted from (or quantities related to) the simulation model (quantities without this superscript are extracted from the real system). Three possible uses of the simulation model data are proposed:

- The simplest use, termed "no reconstruction" provides the simulated science $\mathrm{PSF}$ as the reconstructed PSF, thereby bypassing the previously described LGS and TT reconstruction steps. We find that this approach is inaccurate when the simulation model is slightly detuned (for instance in the Fried parameter) from the real observing conditions.

- The second use of the simulation data, termed "unbalanced extrapolation" employs the simulated LGS and science TTR SFs (these quantities can be directly computed from the simulated residual phase) to perform the LGS-to-science extrapolation step. Note that in order to make this extrapolation step robust against modeling errors in the Fried parameter, we find that it is critical that it be performed in SF rather than in OTF space. The extrapolation filter is thus computed as the ratio of the simulated science TTR SF to the simulated LGS $\mathrm{SF}$, and is applied as a multiplicative filter to the reconstructed system LGS SF. Mathematically, this is expressed as follows:

$$
\hat{D}_{\mathrm{TTR}}^{u}=\hat{D}_{\mathrm{LGS}} \frac{D_{\mathrm{TTR}}^{\mathrm{mod}}}{D_{\mathrm{LGS}}^{\mathrm{mod}}},
$$

where superscript $u$ indicates that it is the so-called "unbalanced" TTR science formula, and subscript "mod" indicates quantities accumulated in simulation from the simulated residual phase, i.e., accumulation of Eq. (2) for the TTR science and LGS phases, i.e., the residual TTR phases for an on-axis point source at infinite and finite range respectively. Multiplication and division in Eq. (15) are performed component-wise (component-wise operations cannot be performed on the covariance matrix since they produce indefinite matrices).

- Finally, the third use of the simulation model, termed "balanced extrapolation," supplements the aforementioned LGS and TT estimation steps with similar steps performed from simulation telemetry, i.e., from simulated measurement covariance matrices. The extrapolation filter is then computed as the ratio of the simulated science TTR SF to the reconstructed simulation LGS SF, i.e., the SF reconstructed from the on-axis LGS WFS measurement 
covariance matrix recorded as part of the simulation telemetry data. Mathematically, this is expressed as in Eq. (15) but with a hat (to indicate an estimated quantity) in the denominator of the SF ratio:

$$
\hat{D}_{\mathrm{TTR}}^{b}=\hat{D}_{\mathrm{LGS}} \frac{D_{\mathrm{TTR}}^{\mathrm{mod}}}{\hat{D}_{\mathrm{LGS}}^{\text {mod }}},
$$

where superscript $b$ indicates that it is the so-called "balanced" TTR science formula, $\hat{D}_{\mathrm{LGS}}^{\mathrm{mod}}$ is obtained following the same procedure that lead to Eq. (12), with $C_{g \mathrm{LGS}}-\hat{C}_{g \mathrm{LGSnse}}$ in Eq. ( $\left.\underline{10}\right)$ replaced by $C_{g \mathrm{LGS}}^{\mathrm{mod}}-$ $\hat{C}_{g \mathrm{LGSnse}}^{\mathrm{mod}}$, i.e., equivalent quantities but retrieved from the simulation model. $C_{g \mathrm{LGS}}^{\mathrm{mod}}$ is directly accumulated in simulation, whereas $\hat{C}_{g \mathrm{LGSnse}}^{\mathrm{mod}}$ is obtained by postprocessing the simulation subaperture average pixel intensities and centroider gains.

Note that for the ideal case of a simulation model perfectly matched to the system, $\hat{D}_{\mathrm{LGS}}=\hat{D}_{\mathrm{LGS}}^{\text {mod }}$, i.e., Eq. (16) provides an exact estimate of the TTR SF, which constitutes an important result since the formula is therefore expected to perform well provided the simulation model does not depart too far off from the real system (quantified in Section 3). We find that, provided the simulation model is sufficiently close to the real system, such an approach relaxes the LGS SF reconstruction accuracy requirements, in particular it eliminates the need for spatial filtering and dealiasing (i.e, these steps can be omitted in the postprocessing of the system and simulated onaxis LGS WFS measurement covariance matrices).

Finally, in order to address the missing term in Flicker's TT OTF formula, balanced extrapolation is also performed on the TT component of the OTF by multiplying Flicker's TT OTF estimate by an extrapolation TT filter, expressed as the ratio of the simulation science TT OTF to the reconstructed simulation science TT OTF. This new filter is defined in OTF space since the ratio of two TT SFs is no longer a TT SF, and the TT Zernike mode covariance matrix is nondiagonal (off-diagonal magnitude can be as large as diagonal values), preventing componentwise operations. Mathematically, this is expressed as follows:

$$
\widehat{\mathrm{OTF}}_{\mathrm{TT}}^{b}=\widehat{\mathrm{OTF}}_{\mathrm{TT}} \frac{\mathrm{OTF}_{\mathrm{TT}}^{\mathrm{mod}}}{\widehat{\mathrm{OTF}}_{\mathrm{TT}}^{\mathrm{mod}}},
$$

where superscript $b$ indicates that it is the so-called "balanced" TT science formula, $\widehat{\mathrm{OTF}}_{\mathrm{TT}}$ is Flicker's (unbalanced) estimate obtained by substituting Eq. (14) into Eq. (7) and then Eq. (9), $\mathrm{OTF}_{\mathrm{TT}}^{\bmod }$ is accumulated in simulation from the simulated TT residual science phase, and $\widehat{\mathrm{OTF}}_{\mathrm{TT}}^{\text {mod }}$ is obtained following the same procedure that lead to $\widehat{\mathrm{OTF}}_{\mathrm{TT}}$, with $C_{g \mathrm{NGS}}-\hat{C}_{g \mathrm{NGSnse}}$ in Eq. (14) replaced by $C_{g \mathrm{mGS}}^{\mathrm{mod}}-$ $\hat{C}_{\text {gNGSnse }}^{\text {mod }}$, i.e., equivalent quantities but retrieved from the simulation model. Equation (17) is equivalently expressed in terms of the TT Zernike mode covariance matrices as follows:

$$
\hat{C}_{\mathrm{TT}}^{b}=\hat{C}_{\mathrm{TT}}+C_{\mathrm{TT}}^{\mathrm{mod}}-\hat{C}_{\mathrm{TT}}^{\mathrm{mod}},
$$

where $C_{\mathrm{TT}}^{\mathrm{mod}}$ denotes the actual simulation TT modal covariance matrix in the science direction of interest, and $\hat{C}_{\mathrm{TT}}^{u, m o d}$ the estimate computed from simulation NGS WFS telemetry data following the same procedure that lead to $\hat{C}_{\mathrm{TT}}$. Equation (18) defines a valid TT modal covariance matrix provided the expression is positive definite. Note that for the ideal case of a simulation model perfectly matched to the system (i.e., $\hat{C}_{\mathrm{TT}}=\hat{C}_{\mathrm{TT}}^{\mathrm{mod}}$ ), Eq. (18) provides an exact estimate of the TT covariance matrix.

We end this section with a note on discretization effects. Since Eqs. (15) and (16) are computed at coarse resolution $\left(\overline{\Delta x}=d_{\mathrm{SA}}\right)$, the DM fitting and aliasing errors are underestimated. In order to correct for this error, the following procedure should be followed: (i) bilinearly up-sample Eq. (8) to a fine resolution, $\Delta x_{0} / \lambda \ll \Delta x / \lambda$, (ii) transform to "log OTF" space by taking the negative logarithm of the bilinearly up-sampled reconstructed OTF divided by the diffraction limited OTF, (iii) multiply by a precomputed filter, (iv) inverse transform to return to OTF space. Such a procedure also provides a means to properly extrapolate the reconstructed PSF Eq. (4) to a large FoV equal to $\lambda / \Delta x_{0}$. The filter (which depends mainly on the DM influence functions) is precomputed by an offline AO simulation, and is expressed as a log OTF ratio, whose numerator is computed from the accumulated residual phase sampled at fine resolution $\Delta x_{0}$, and denominator from the bilinearly up-sampled OTF resulting from the accumulated residual phase sampled at the reconstruction resolution $\Delta x=d_{\mathrm{SA}}$. As illustrated in Fig. 4, such a filter is independent of the value of the Fried parameter since the log OTF is essentially an aperture-averaged $\mathrm{SF}$, and an SF ratio is insensitive to seeing, which is not the case for a simple minded OTF ratio (Fried parameter dependence of the numerator and denominator OTFs does not cancel out, in fact shift-invariant SFs subtract in an OTF ratio). All simulation results presented in Section 3 are given for simulated PSFs accumulated from residual phases coarsely sampled at the reconstruction resolution; hence this additional filtering step has not been implemented.

\section{Performance Evaluation}

In order to evaluate the accuracy of the LGS MCAO PSFR algorithm defined in Section 2, a set of 50,000 step simulations were performed on a pair of GTX 580 Graphics Processing Units using the in-house developed Multithreaded Adaptive Optics Simulator $[23,24]$. The TMT LGS MCAO system under design was simulated to high fidelity, and the achieved simulation speed was $100 \mathrm{~ms} / \mathrm{step}$ (i.e., 2 orders of 

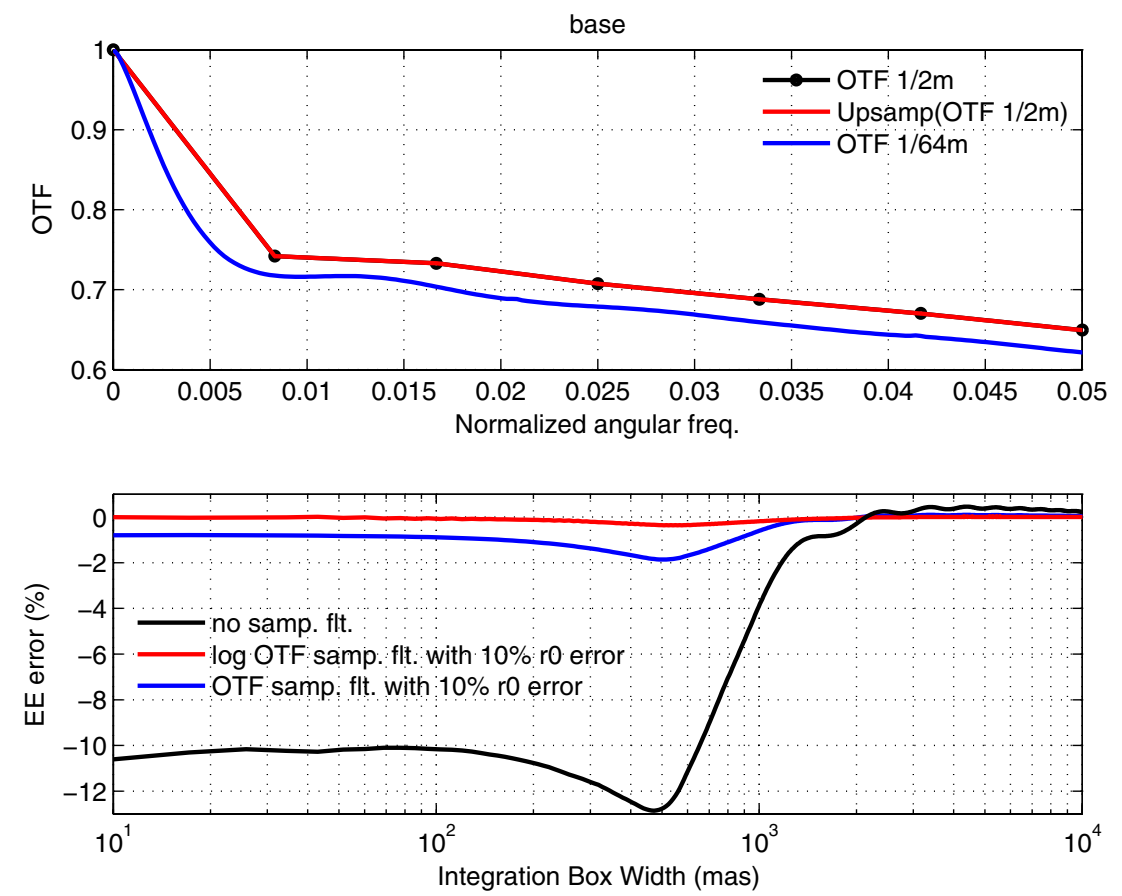

Fig. 4. (Color online) Illustration of OTF reconstruction errors introduced by discretization effects. Top panel shows simulated J-band TMT LGS MCAO OTFs obtained by sampling the residual aperture-plane phase either at $1 / 2 \mathrm{~m}$ or at $1 / 64 \mathrm{~m}$. Bottom panel shows the EE error introduced by the coarse sampling (black curve, leads 10\%-12\% error), as well as the reconstruction error of two procedures to recover the finely sampled OTF from the coarsely sample one in the presence of a $10 \% r_{0}$ modeling error, one operating on the OTF (second line, leads $1 \%-2 \%$ error), the other on the logarithm of the OTF (top line, leads $0.1 \%$ error).

magnitude slower than real-time), which includes $\mathrm{PSF}$ and covariance matrix computations on the $D_{0}=30 \mathrm{~m}$ aperture-plane grid sampled at $\Delta x=$ $d_{\mathrm{SA}}=1 / 2 \mathrm{~m}$. PSFR accuracy has been assessed by computing $J$-band EE error curves, $(\mathrm{EE}-\widehat{\mathrm{EE}}) / \mathrm{EE}$, whose values near the origin correspond to the SR error. The analysis has been performed over a 30 arcsec diameter science FoV. The seven-layer turbulence/ wind profile shown in Table 1 has been simulated.

The profile was obtained from measurements performed during the three-year TMT site testing campaign on Mauna Kea [25]. The outer scale at each layer is $L_{0}=30 \mathrm{~m}$. Two profile strengths were simulated: a baseline strength $r_{0}$ of $18.6 \mathrm{~cm}$ (corresponding to median Mauna Kea seeing, i.e., 0.55 arcsec at $500 \mathrm{~nm}$ ), and a $09 \mathrm{r} 0$ strength of $16.7 \mathrm{~cm}$ (corresponding to roughly 65 percentile Mauna Kea seeing, i.e. 0.62 arcsec at $500 \mathrm{~nm}$ ). The profile isoplanatic angle is 2.2 arcsec, and the $2 \mathrm{DM}$ generalized isoplanatic angle [16] for a DM conjugation range of $11.2 \mathrm{~km}$ is 8.2 arcsec. The Greenwood frequency is $22 \mathrm{~Hz}$. All these atmospheric turbulence parameters are quoted at a reference wavelength of $500 \mathrm{~nm}$.

The system implements separate high-order LGS and low-order NGS servo loops [26]. The LGS loop runs a tomographic minimum variance wavefront

Table 1. Turbulence Profile Relative Weights and Wind Speed

\begin{tabular}{lccllrrr}
\hline Altitude $(\mathrm{km})$ & 0 & 0.5 & 1 & 2 & 4 & 8 & 16 \\
Wind Speed $(\mathrm{m} / \mathrm{s})$ & 5.6 & 5.8 & 6.2 & 7.6 & 13 & 19 & 12 \\
Weights $(\%)$ & 46 & 13 & 4 & 5 & 12 & 9 & 11 \\
\hline
\end{tabular}

reconstruction algorithm at $800 \mathrm{~Hz}$ from measurements from six high-order LGS Shack-Hartmann WFSs (each of order $60 \times 60$ ). The NGS loop runs a classical least-squares reconstruction matrix, $R_{\mathrm{NGS}}$, at low frame rate (generally around $90 \mathrm{~Hz}$ ), controlling five modes, defined as global TT and three TTA modes distributed on the system's two DMs, from the combined measurements from a tip/tilt/focus/ astigmatism (TTFA) NGS WFS (i.e., an order $2 \times 2$ Shack-Hartmann WFS) and two additional fullaperture TT NGS Shack-Hartmann WFSs [15]. The TTFA NGS WFS is required in order to disentangle the atmospheric and mesospheric sodium layer focus errors. Such a low-order NGS loop is required in order to minimize image distortion (absolute and differential magnification) due to residual atmospheric TT jitter across an extended FoV.

Regarding the servos, the LGS loop implements a simple type I servo with a gain of $50 \%$, whereas the NGS loop uses a more sophisticated type II servo, whose parameters (sampling frequency, modal gains and lead filter cross-over frequency, and phase margin parameters) are obtained from an optimization procedure performed by a separate highfidelity sky coverage simulation tool $[\underline{27}, \underline{28}]$. Sample NGS loop theoretical error rejection curves are plotted in Fig. 5. Each curve is given by $10 \log _{10}\left(\left|1 /\left(1+H_{\mathrm{OL}}\right)\right|^{2}\right)$, where $H_{\mathrm{OL}}$ denotes the open-loop transfer function of the NGS loop (product of WFS, servo lag, digital-to-analog conversion, cascaded integrators and lead filter transfer functions [29]), and illustrates the characteristic $-40 \mathrm{~dB}$ 


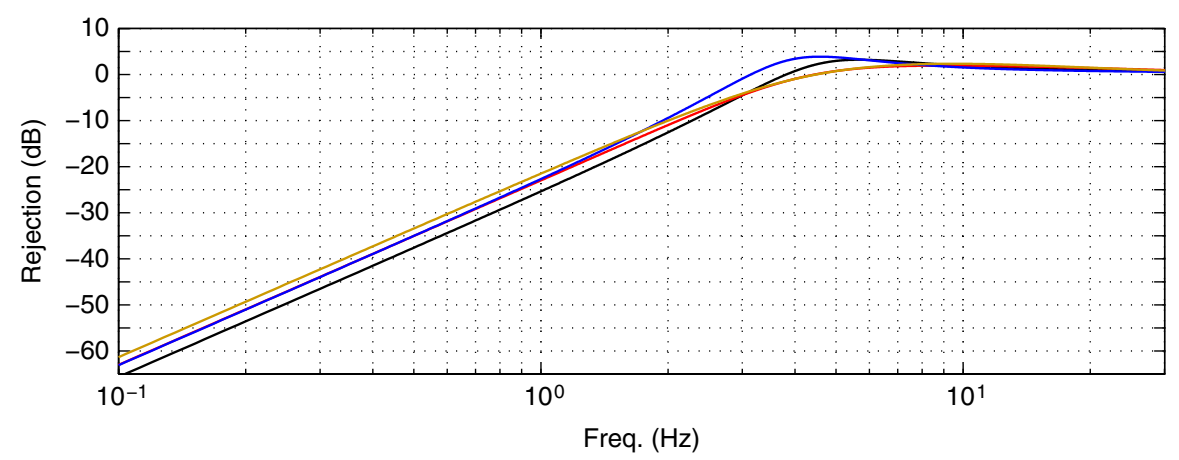

Fig. 5. (Color online) Sample error rejection curves for the NGS type II servo loop.

rejection per decade below the $\sim 3 \mathrm{~Hz}-3 \mathrm{~dB}$ rejection bandwidth. The four different curves correspond to four different sets of servo parameters investigated in this paper (each optimized for a particular NGS asterism).

Finally, each LGS WFS subaperture is imaged by an array of up to $16 \times 6$ detector pixels [500 milliarcseconds (mas) pixel scale on the sky], whose intensities are converted into subaperture average phase gradient by linear matched filter weights [18], whereas each NGS WFS subaperture is imaged by $4 \times 4$ detector pixels (Nyquist sampled in $\mathrm{H}$ band, i.e., 5.7 mas pixel scale on the sky for the 2 TT WFSs and 11.4 mas pixel scale for the TTFA WFS), whose intensities are similarly converted into average phase gradient by linear matched filter weights. All these features are fully integrated in the simulation model. Readout noise was simulated at the level of three electrons RMS per read per pixel, both for the high-order LGS and low-order NGS WFSs. Two distinct LGS WFS signal levels were simulated: 900 (baseline) and 720 (08pde) photo-detected electrons per subaperture per sampling period [30].

The accuracy of the PSFR scheme has been assessed by assigning some of the data to the "real system," some to the "simulation model," and performing the postprocessing steps described in Section 2. Namely, we have considered the following four (system, model) combinations: (09r0, 09r0), i.e., perfectly matched simulation and system, both in 09r0 turbulence, (09r0, base), i.e., system in 09r0 turbulence, model in baseline turbulence, (jit, base), i.e., system and model in baseline turbulence with system impacted by an unmodeled $2 \mathrm{~Hz}, 3$ mas RMS TT jitter, and (08pde, base), i.e., system and model in baseline turbulence with system impacted by an unmodeled $20 \%$ LGS WFS signal reduction on all LGS WFSs. For each of these four cases, PSFR accuracy has been broken down into individual TTR and TT reconstruction errors, and total reconstruction error, which includes the two fundamental PSFR errors: TTR/TT decoupling approximation and long-exposure approximation. The $(09 \mathrm{r} 0,09 \mathrm{r} 0)$ matched system and model scenario is of interest to assess the accuracy of the unbalanced TTR and TT reconstructions, as well as that of the fundamental TT/TTR decoupling and long exposure approximations.
We have performed the PSFR performance evaluation study for four different NGS asterisms. Constellation geometries and star magnitudes are shown in the top panels of Fig. 6. Each asterism consists of a TTFA and two TT stars. The black constellation (asterism 1) is an ideal symmetrical asterism of stars of equal magnitude (19 in. $\mathrm{J}$ band) on a 30 arcsec radius circle. The red, blue, and brown constellations (asterisms $2,3,4$ ) are asymmetrical (all within a $1 \mathrm{ft}$. radius NGS patrol field) and of stars of different magnitude. Sampling frequencies are respectively $100,80,90$, and $66 \mathrm{~Hz}$ (result of an optimization procedure performed by a separate high-fidelity sky coverage simulation tool $[27,28])$. Bottom panels display the signal-to-noise ratio (SNR) of each WFS (TTFA, TT, TT ordering), as well as the RMS noise equivalent angle in units of mas (NEA, i.e. the square root of the trace of $\hat{C}_{g N G S n s e}$ ) at the sampling frequency of the loop. To give a point of comparison, for $D_{0}=30 \mathrm{~m}$, the diffraction limited $J$ band PSF full-width at half maximum (FWHM) is $\lambda / D_{0} \sim 8.6$ mas, and the TT WFSs pixel scale is 5.7 mas ( $\mathrm{H}$ band Nyquist sampling). A $40 \%$ end-to-end optical throughput (telescope $+\mathrm{AO}+$ instrument) has been assumed to compute the SNR.

Figure 7 displays NGS loop performance during a $\sim 50 \mathrm{~s}$ exposure time. Performance is given in terms of cumulative NGS modes (i.e., global TT and three TTA modes) and TT mode WFE averaged over a $17^{\prime \prime} \times 17^{\prime \prime}$ FoV. Performance has been averaged over five uncorrelated realizations of atmospheric frozen flow turbulence. The plots are indicators of NGS loop stability and stationarity. The initial transients observed in some of the curves disappear once the averaging time exceeds a few seconds. The cumulative curves are otherwise very stable and flat, indicating that the NGS loop is stable and stationary for all $4 \times 4$ cases investigated. The jitter case refers to the baseline condition with an additional injected TT jitter sine wave at $2 \mathrm{~Hz}$ and of an RMS value equal to $\sigma_{\theta}=3$ mas angular displacement on the sky, i.e., a WFE of $\sigma_{\mathrm{TT}}=D_{0} \sigma_{\theta} / 4 \sim 109 \mathrm{~nm}$. As seen from Fig. 5, the NGS type II servo loop rejects this disturbance to $\sim-10 \mathrm{~dB}$ in variance (i.e. $\sim 68 \%$ in. RMS).

We start by discussing PSFR accuracy for the on-axis direction, i.e., the center of the science FoV. 

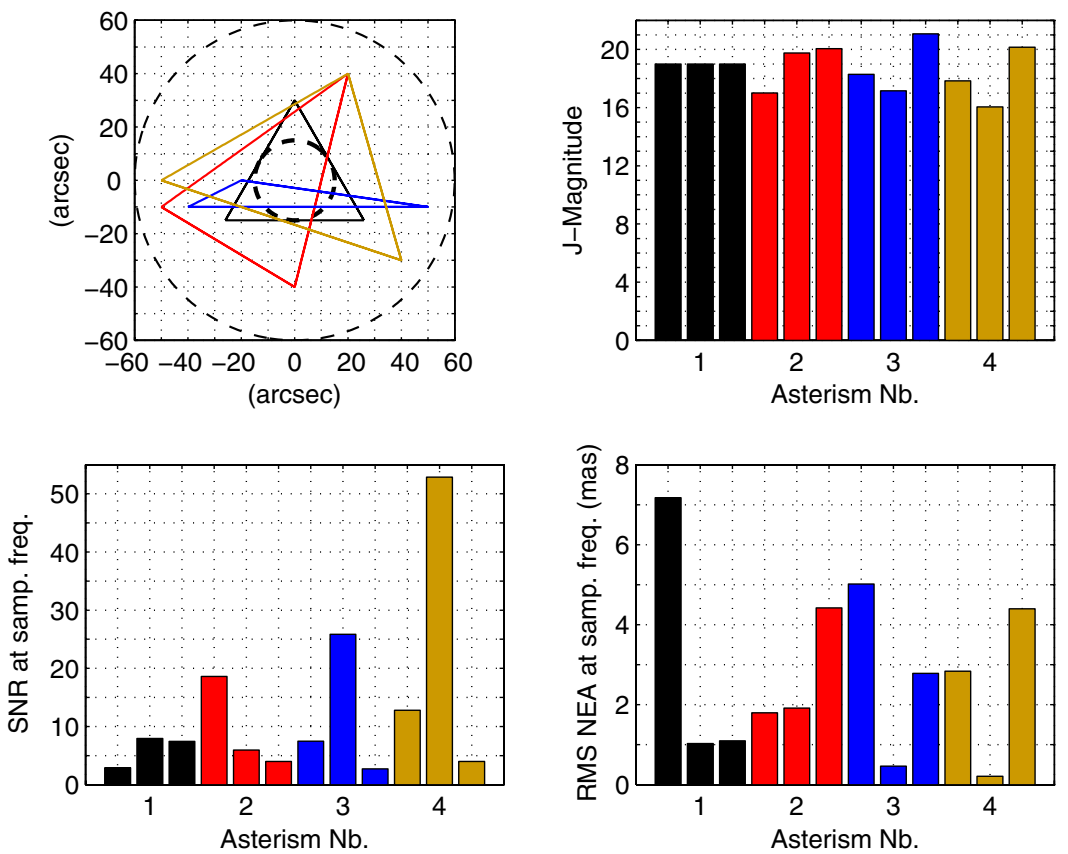

Fig. 6. (Color online) Top: geometry and star brightness of four sample NGS asterisms investigated in this paper. Outer circular dashed boundary indicates the 2 arcmin diameter NGS patrol FoV, whereas the inner dashed circle indicates the 30 arcsec diameter science FoV. Bottom: signal-to-noise ratio and RMS noise equivalent angle.

Results are broken down into TTR, TT, and total EE reconstruction error. The total error includes the TTR and TT reconstruction errors as well as the fundamental decoupling error (OTF estimated as the product of TTR and TT OTFs) and the long-exposure approximation. The following line styles have been adopted throughout the paper: dotted lines are EE errors for PSFs generated directly by the simulation model without any postprocessing of system or simulation WFS telemetry (the "no reconstruction" approach), solid lines are errors for PSFs estimated using "unbalanced extrapolation," and dashed lines are errors for PSFs estimated using "balanced extrapolation.” Line colors follow Fig. $\underline{7}$ 's legend.
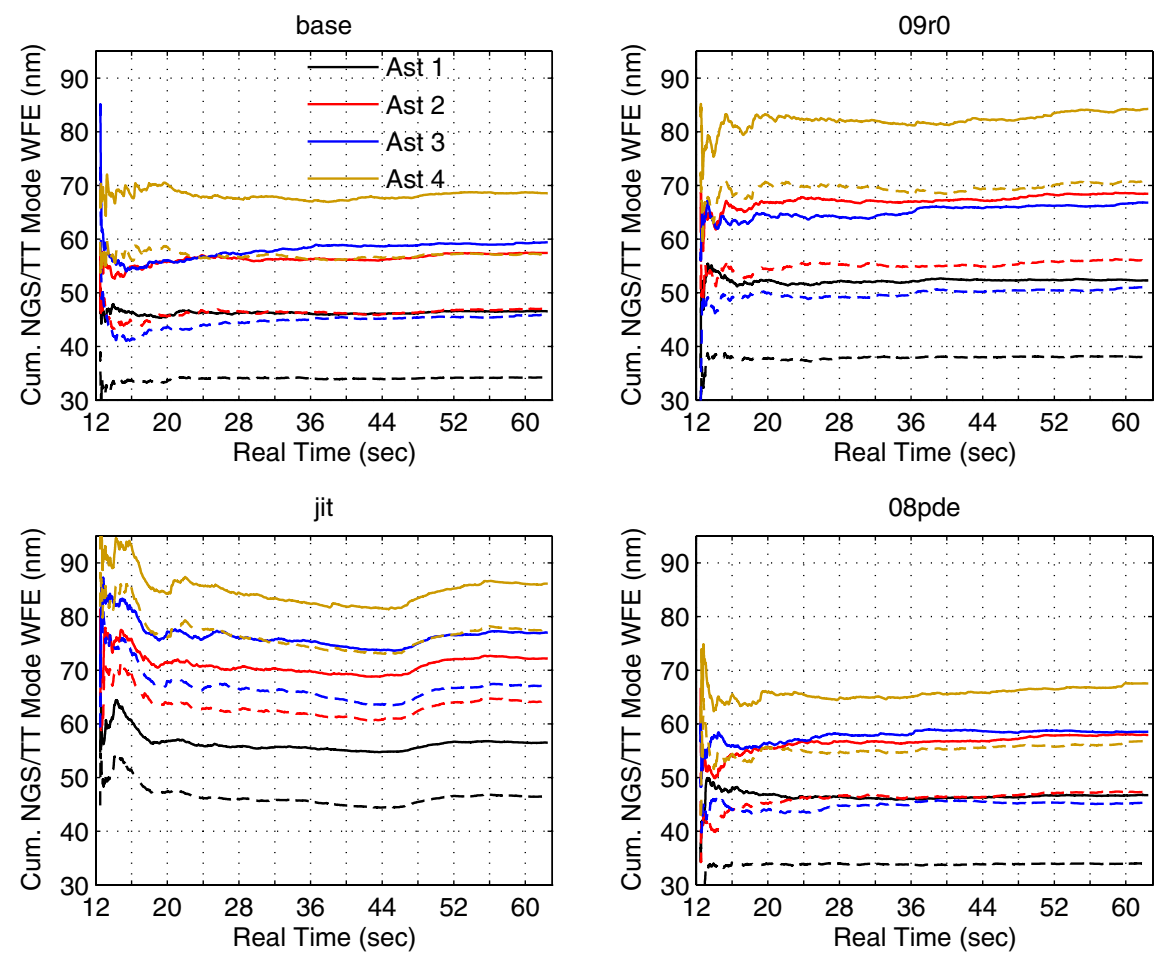

Fig. 7. (Color online) Cumulative NGS (solid) and TT (dashed) mode WFE averaged over a 17" × 17" FoV for the baseline, 09r0, jitter and 08pde observing conditions. See text for details. 

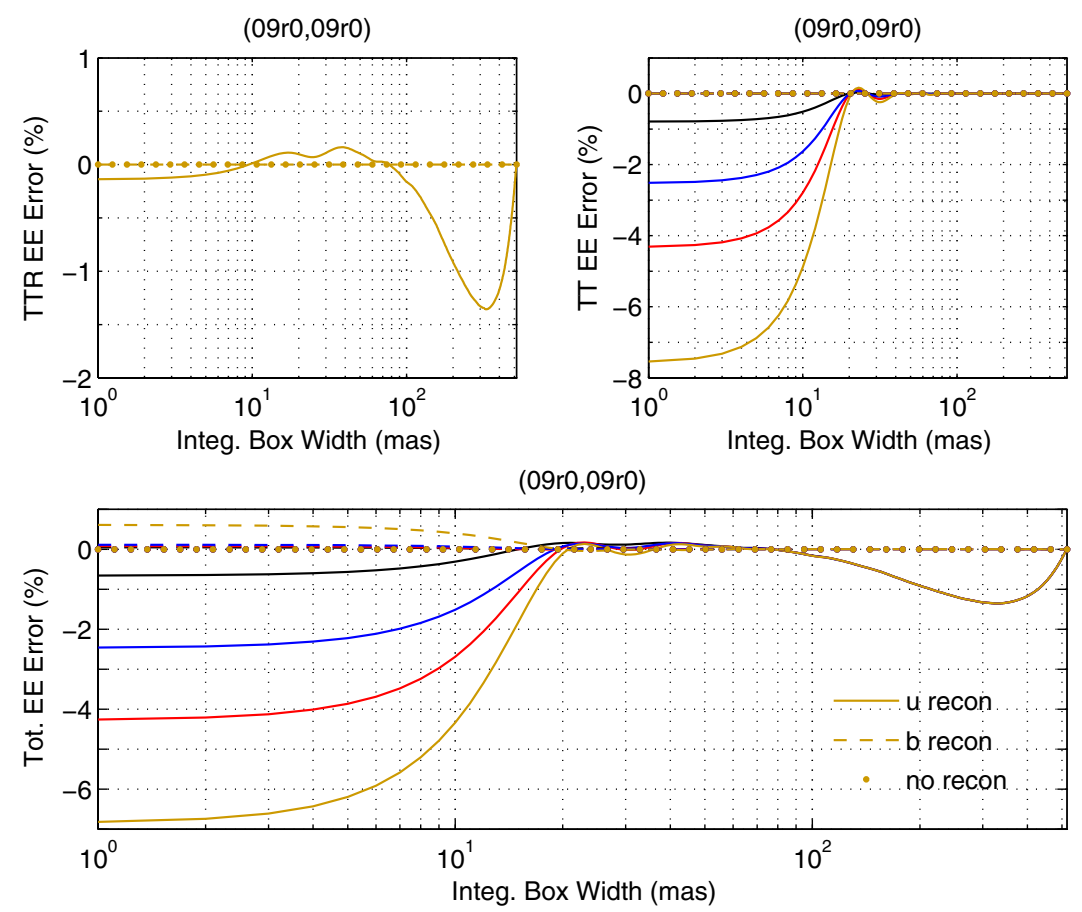

Fig. 8. (Color online) On-axis PSF EE reconstruction error for the ideal case of a simulation model perfectly tuned to the system. See text for details.

Figure 8 illustrates sample results for the ideal case of a simulation model perfectly matched to the system. Such a scenario is clearly unrealistic, but of importance to assess the TTR and TT reconstruction errors for unbalanced extrapolation (balanced extrapolation and the no reconstruction approach yielding both exact TTR and TT reconstructions in such a case).

Salient features are the following:

(1) In order to yield the science TTR PSFR error curve displayed in solid in the upper left panel, an ad hoc Laplacian squared (also known as biharmonic) regularization matrix with $\sim 25 \%$ weight relative to the largest eigenvalue of $\Gamma^{T} \Gamma$ had to be included in the computation of $\left(\Gamma^{T} \Gamma\right)^{\dagger}$ in Eq. (11). The necessity to include this regularization term is not fully understood, but is believed to be related to the presence of wafflelike modes originating from the ShackHartman Fried geometry. Although the few eigenvalues in the null space of $\Gamma^{T} \Gamma$ are clustered 12 orders of magnitude below the rest of the spectrum (which spans 3 orders of magnitude), applying a truncated singular value decomposition to $\Gamma^{T} \Gamma$ does not solve this problem, regardless of the thresholding level. Without regularization, the SR error is $\sim 30 \%$, it drops to $\sim 5 \%$ for a $15 \%$ regularization weight (38 mas RMS) and to $\sim 0 \%$ for a $25 \%$ weight (49 mas RMS). We have verified that similar error levels occur for the ideal case of noise-free, fully linear Shack-Hartmann WFSs. Note that there is still a $\sim 1.5 \% \mathrm{EE}$ error in the PSF tail for an integration window of width $\sim 0.25 \lambda / r_{0} \sim 350$ mas, likely related to inaccurate reconstruction of residual aliasing and fitting error.
(2) The TT error (which is strongly NGS asterism dependent) is negative, i.e., the estimated SR is too high (estimated TT covariance matrix is too weak). The fact that Flicker's formula, Eq. (14), does not generally do a good job at estimating the science TT covariance matrix suggests that it misses a TTA term or a high-order LGS-uncorrected atmospheric turbulence aliasing term or possibly a combination of both. This missing term is captured in the balanced extrapolation formula Eq. (18) as will be seen further below. We should also point out that the simulation results discussed by Flicker et al. in [17] are somewhat oversimplified since (i) the simulated atmosphere consisted of only TT and quadratic modes, and (ii) NGS WFSs were modeled using a geometrical linear model (infinite dynamic range).

(3) The TT error is concentrated in the PSF core (in an area of size equal to roughly twice the PSF FWHM), and gradually degrades between the different asterisms following Fig. 7's NGS loop performance degradation. The Maréchal approximation relating SR loss to TT WFE, $\sigma_{\theta} /\left(\lambda / D_{0}\right) \sim$ $(2 / \pi) \sqrt{-\ln \left(\mathrm{SR}_{\mathrm{TT}}\right)}$, indicates that in order to yield a SR estimation error of respectively $1 \%, 2 \%$, and $3 \%$, the RMS TT estimation error, $\sigma_{\theta}$, has to be respectively equal to $6 \%, 9 \%$, and $11 \%$ of the diffraction limited PSF FWHM, $\lambda / D_{0}$, which places a challenging requirement on the TT reconstruction algorithm.

(4) The end-to-end total (final) reconstruction error (which includes the decoupling and long exposure error), is largely dominated by the TT reconstruction error and to a smaller extent by the footprint of the LGS PSF reconstruction error in the PSF tails. The small, nonzero value of the total error for the 

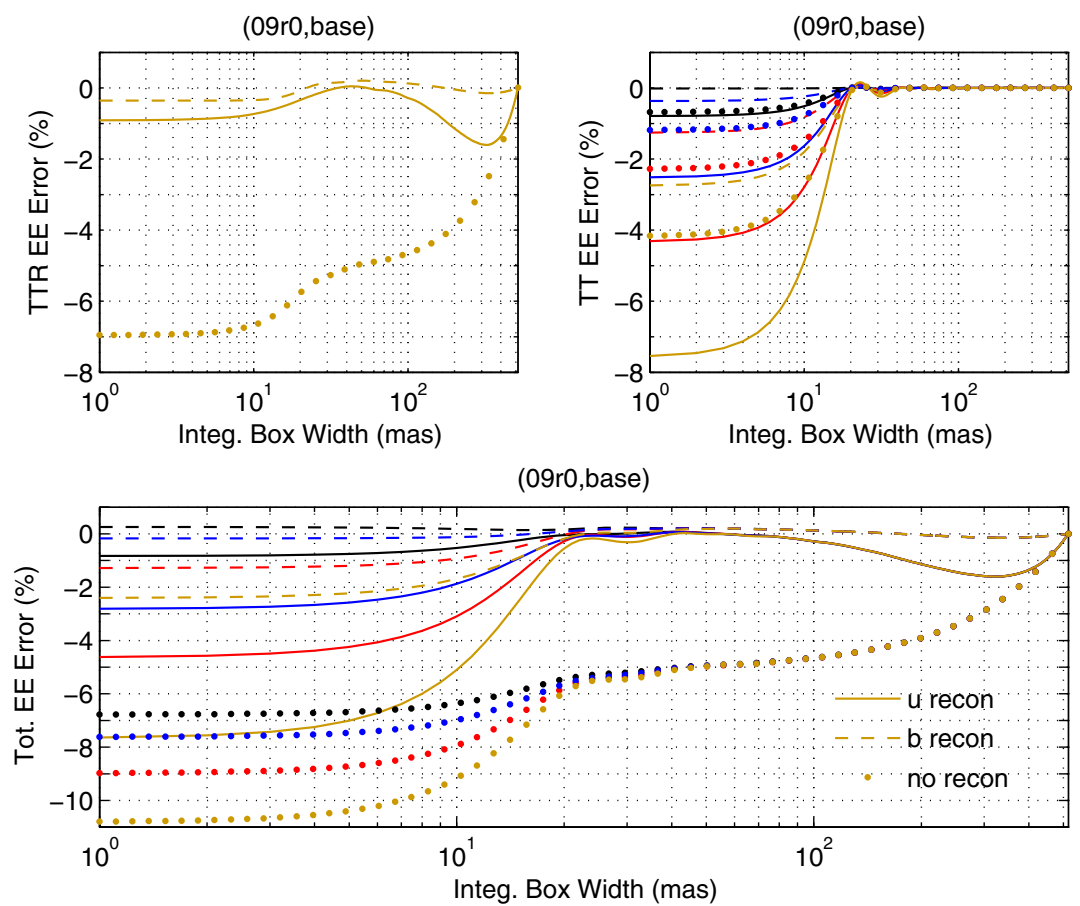

Fig. 9. (Color online) Sample PSF reconstruction accuracy for the case of a simulation model detuned from the system by a $10 \%$ Fried parameter error. See text for details.

balanced formula quantifies the magnitude of the decoupling and long exposure errors, which is negligible for the four NGS asterisms investigated in this paper.

Figures $\underline{9}, \underline{10}$, and $\underline{11}$ assess PSFR accuracy for the case of a simulation model detuned from the system by respectively a 10\% Fried parameter error, an unmodeled $2 \mathrm{~Hz}, 3$ mas TT jitter, and a 20\% LGS WFS signal level error (the same model simulating the baseline observing condition has been adopted for all three cases). For the balanced extrapolation Eq. (16), the LGS reconstruction matrix Eq. (11) does not need to incorporate regularization, and the aliasing term, $\hat{C}_{\text {alias }}$, can be excluded from both $\hat{D}_{\text {LGS }}$ and
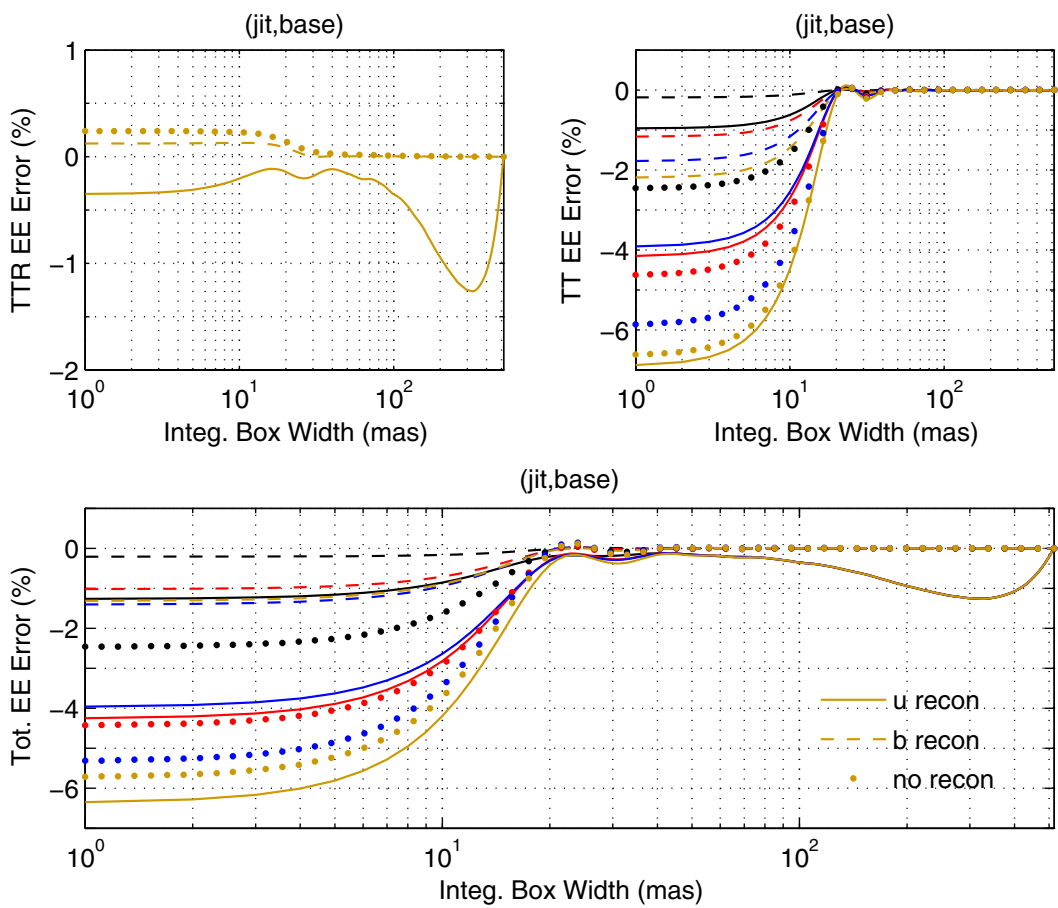

Fig. 10. (Color online) Sample PSF reconstruction accuracy for the case of a simulation model detuned from the system by an unmodeled $2 \mathrm{~Hz} 3$ mas TT jitter. See text for details. 

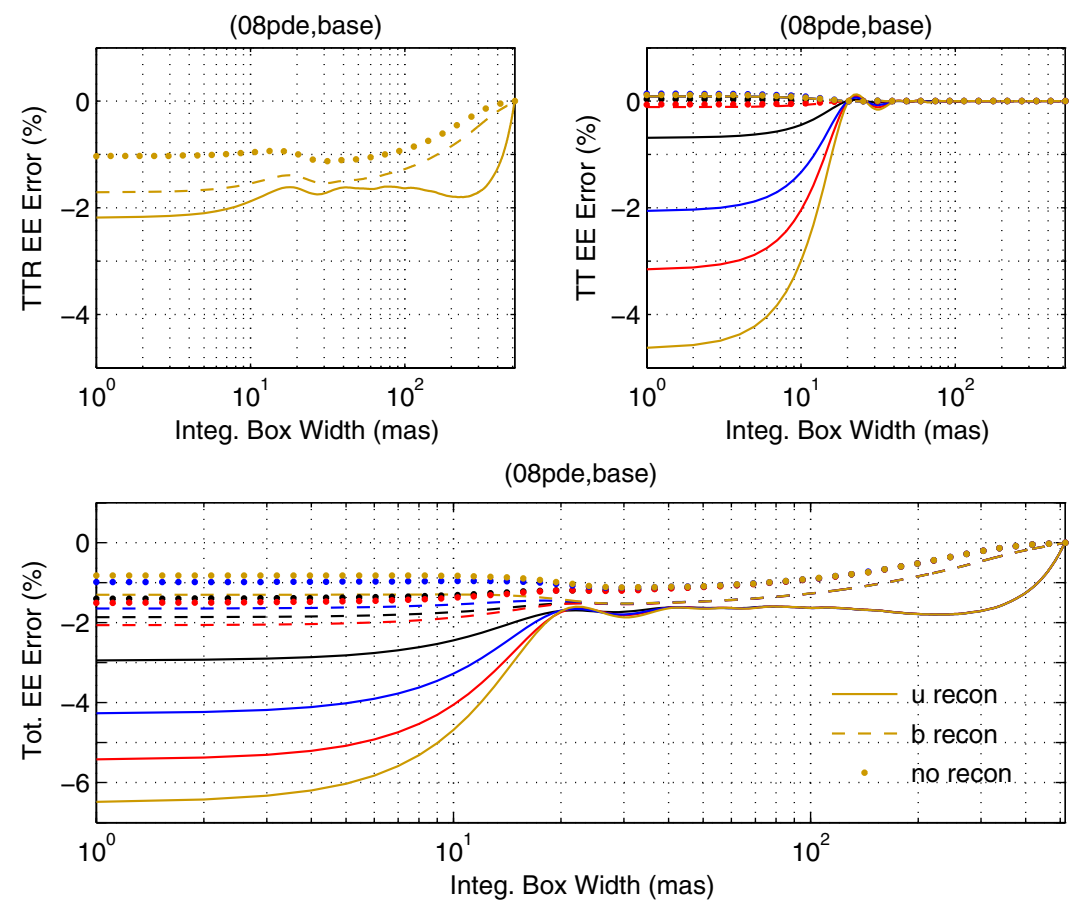

Fig. 11. (Color online) Sample PSF reconstruction accuracy for the case of a simulation model detuned from the system by a $20 \%$ LGS WFS signal level error. See text for details.

$\hat{D}_{\mathrm{LGS}}^{\mathrm{mod}}$. Although this leads to large LGS PSFR errors for the system and the model, these errors cancel out in the SF ratio, and the science TTR PSFR is accurate. Salient features for the case of a simulation model detuned in seeing are the following:

(1) The TTR PSFR error is robust against such errors, a result that applies to both unbalanced and balanced extrapolation. The balanced formula provides superior reconstruction quality since it eliminates the LGS reconstruction error in the PSF tail.

(2) The TT error for Flicker's formula shows the same pathology that was discussed in Fig. 8 because the formula is model independent and the system is in the same observing condition as in Fig. 8 .

(3) The TT error for the balanced approach is significantly reduced compared to the error for the unbalanced approach: three out of the four NGS asterisms investigated yield subpercent TT SR errors.

(4) The end-to-end reconstruction error for the balanced approach is at the subpercent level for three out of the four NGS asterisms investigated, and entirely concentrated in an SR error.

Salient features for the case of an unmodeled TT jitter are the following:

(1) The TTR error for the balanced approach is robust against such errors, yielding essentially no reconstruction error.

(2) The TT error for the balanced approach is significantly smaller than the error for the unbalanced approach, but a residual SR error remains, whose magnitude is at or above the percent level.
(3) The end-to-end reconstruction error for the balanced approach is entirely dominated by the TT error for the four asterisms investigated.

Finally, salient features for the case of a $20 \%$ LGS WFS signal level modeling error are the following:

(1) There is a conflicting behavior between the science and LGS PSFs for a modeling error of this type: while the science SR is degraded by a LGS WFS signal level reduction, the LGS SR actually improves, thereby leaving a nonzero residual error in the SF ratios, which translates into TTR SF errors. This is clearly observed in Fig. 11, where both

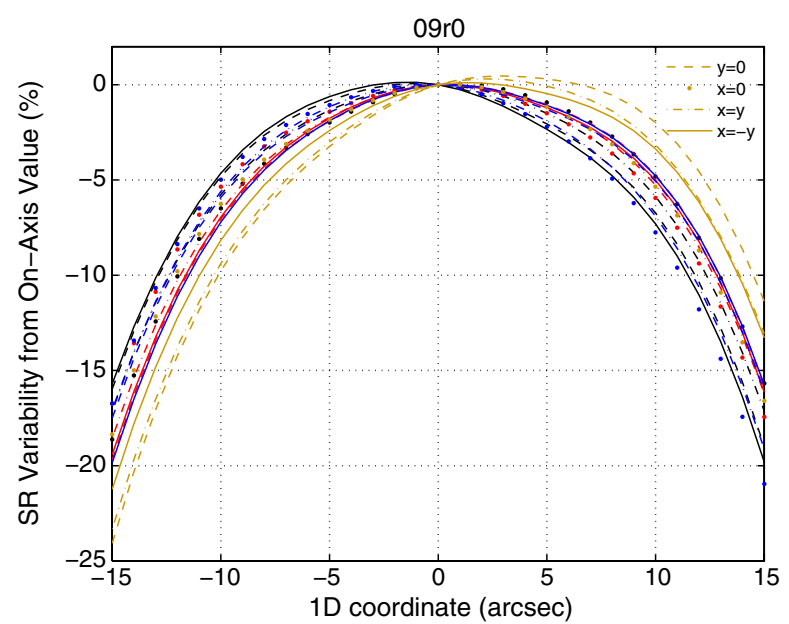

Fig. 12. (Color online) J-band science SR variability from on-axis value, along the $30 \mathrm{in}$. wide $x=0, y=0, x=y$ and $x=-y$ field point lines. 

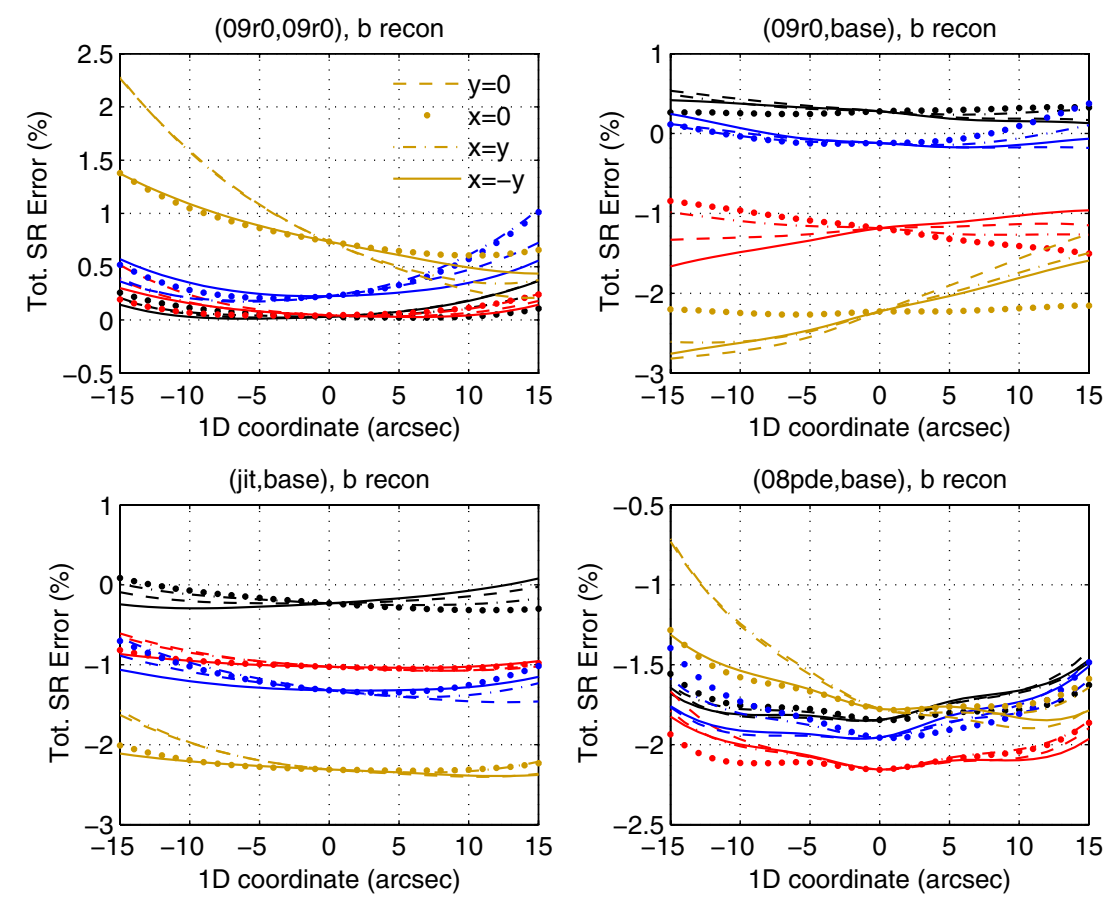

Fig. 13. (Color online) Total J-band SR error for the balanced approach along the 30 in. wide $x=0, y=0, x=y$ and $x=-y$ field point lines. Upper left panel shows the error due to the decoupling and long-exposure approximations for the 09r0 observing condition. Upper right, lower left, and lower right panels show respectively the total error for a simulation model with a $10 \%$ Fried parameter error, an unmodeled $2 \mathrm{~Hz}, 3$ mas TT jitter, and a 20\% LGS WFS signal level error.

unbalanced and balanced formulas amplify the error made by relying exclusively on the simulation model (dotted curve). Accurate LGS WFS signal level estimation is thus important to minimize the error induced by the SF ratio for such detunings.
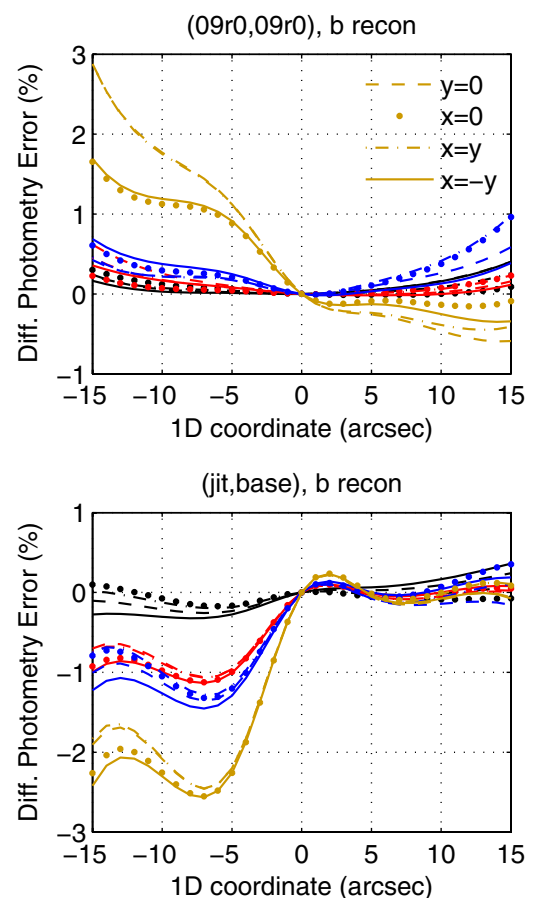

(2) The end-to-end reconstruction error for the balanced approach is entirely dominated by the TTR error.

The remaining figures of the paper assess J-band absolute and relative photometry errors over a 30
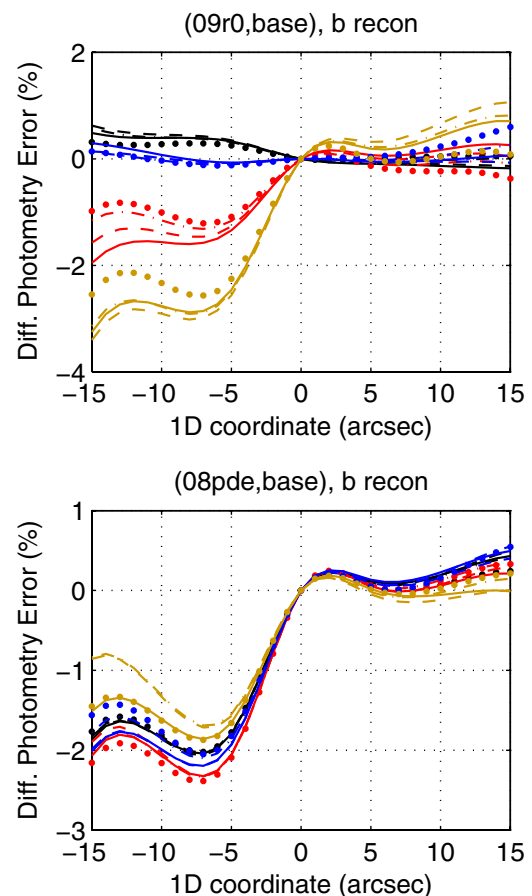

Fig. 14. (Color online) Total J-band differential photometry error for the balanced approach, evaluated with respect to on-axis Strehl along the 30 in. wide $x=0, y=0, x=y$ and $x=-y$ field point lines. Same color and notation conventions as in Fig. 13. 
arcsec diameter circular FoV. Figure 12 shows the relative SR variability across such a field with respect to the on-axis Strehl value, i.e., $100\left(\mathrm{SR}-\mathrm{SR}_{0}\right) / \mathrm{SR}$. Variations typically range from $15 \%-20 \%$ at the edge of the field. Figure 13 illustrates the absolute photometry error, i.e., $100(\mathrm{SR}-\widehat{\mathrm{SR}}) / \mathrm{SR}$, for the balanced approach, along the $30 \operatorname{arcsec}$ wide $x=0, y=0, x=y$ and $x=-y$ field point lines for the cases of (i) a simulation model perfectly matched to the system, (ii) a simulation model with a $10 \%$ Fried parameter error, (iii) an unmodeled $2 \mathrm{~Hz}, 3$ mas TT jitter, and (iv) a $20 \%$ LGS WFS signal level error. Case (i) indicates that the error due to the decoupling and longexposure approximations are below the percent level for asterisms 1-3, and can reach $2 \%$ for asterism 4 . Cases (ii)-(iv) indicate that errors are mostly uniform across the FoV and tend to slightly increase at the edges. Figure 14 displays the relative photometry error, i.e., $100\left(\mathrm{SR} / \mathrm{SR}_{0}-\mathrm{SR} / \mathrm{SR}_{0}\right)$, for the balanced approach. The error is below 3\% for all cases investigated.

\section{Conclusions}

We have discussed an innovative simulation model based approach for atmospheric PSFR in the context of LGS MCAO. The approach is inspired from the classical PSFR scheme developed by Véran et al. in 1997 [10] and Flicker et al. in 2003 [17], and consists in using a simulation model to (i) postprocess a single high-order LGS WFS measurement covariance matrix to compute a TTR OTF for the science target at infinite range, (ii) postprocess the global measurement covariance matrix of the multiple low-order NGS WFSs controlling TT and TTA to compute a TT OTF, and (iii) multiply both OTFs. WFS telemetry postprocessing is performed identically on both system and simulation in order to "balance" the algorithm, thereby enhancing its robustness and accuracy. Sample high-fidelity Monte Carlo simulations for the TMT LGS MCAO system indicate that percent level absolute and differential photometry over moderate fields of views are achievable, provided the simulation model faithfully represents the real system. Sensitive model parameters include overall Fried parameter ( $\sim 10 \%$ accuracy required), LGS WFS signal level ( $20 \%$ accuracy required) and TT jitter.

The authors acknowledge the detailed and thought out comments made by the anonymous reviewers of this paper that undoubtedly lead to an improved presentation of the subject matter. L. Gilles acknowledges Andrei Tokovinin's comments and suggestions. The authors gratefully acknowledge the support of the Thirty Meter Telescope partner institutions. They are the Association of Canadian Universities for Research in Astronomy (ACURA), the California Institute of Technology, the University of California, the National Astronomical Observatory of Japan, the National Astronomical Observatories of China and their consortium partners, and the Department of
Science and Technology of India and their supported institutes. This work was supported as well by the Gordon and Betty Moore Foundation, the Canada Foundation for Innovation, the Ontario Ministry of Research and Innovation, the National Research Council of Canada, the Natural Sciences and Engineering Research Council of Canada, the British Columbia Knowledge Development Fund, the Association of Universities for Research in Astronomy (AURA), and the U.S. National Science Foundation.

\section{References}

1. P. B. Cameron, M. C. Britton, and S. R. Kulkarni, "Precision astrometry with adaptive optics," Proc. SPIE 7015, 70150A (2008).

2. P. B. Cameron, M. C. Britton, and S. R. Kulkarni, "Precision astrometry with adaptive optics," Astron. J. 137, 83-93 (2009).

3. C. Schödel, "Accurate photometry with adaptive optics in the presence of anisoplanatic effects with sparsely sampled PSF: the Galactic center as an example of a challenging target for accurate AO photometry," Astron. Astrophys. 509, 1-16 (2010).

4. R. Davies, H. Engel, E. Hicks, N. F. Schreiber, R. Genzel, L. Tacconi, F. Eisenhauer, and S. Rabien, "Dissecting galaxies with adaptive optics," Proc. SPIE 7736, 77361G (2010).

5. M. Ammons, E. Bendek, and O. Guyon, "Microarcsecond relative astrometry from the ground with a diffractive pupil," Proc. SPIE 8151, 81510T (2011).

6. G. Herriot, D. Andersen, J. Atwood, C. Boyer, A. Beauvillier, P. Byrnes, R. Conan, B. Ellerbroek, J. Fitzsimmons, L. Gilles, P. Hickson, A. Hill, K. Jackson, O. Lardière, J. Pazder, T. Pfrommer, V. Reshetov, S. Roberts, J.-P. Véran, L. Wang, and I. Wevers, "NFIRAOS: TMT"s facility adaptive optics system," Proc. SPIE 7736, 77360B (2010).

7. B. Ellerbroek, S. Adkins, D. Andersen, J. Atwood, S. Browne, C. Boyer, P. Byrnes, K. Caputa, R. Conan, R. Cousty, D. Erikson, J. Fitzsimmons, F. Gamache, L. Gilles, G. Herriot, P. Hickson, O. Lardiere, P. Morin, J. Pazder, T. Pfrommer, D. Quinn, V. Reshetov, S. Roberts, J.-C. Sinquin, M. Schoeck, M. Smith, G. Tyler, J. Vaughn, J.-P. Véran, C. Vogel, L. Wang, and I. Wevers, "First light adaptive optics systems and components for the Thirty Meter Telescope," Proc. SPIE 7736 773604 (2010).

8. L. Jolissaint, C. Neyman, J. Christou, P. Wizinowich, and L. Mugnier, "First successful adaptive optics PSF reconstruction at W. M. Keck observatory" (2012) http://arxiv.org/abs/1202.3486.

9. F. Rigaut, B. Neichel, C. Dorgeville, D. Gratadour, M. Boccas, G. Trancho, M. Bec, C. Trujillo, M. Edwards, and R. Carrasco, "GeMS sees star light," presented at Adaptive Optics for Extremely Large Telescopes II, Victoria, BC, Canada, 25-30 Sept. 2011), http://ao4elt2.lesia.obspm.fr/sites/ao4elt2/IMG/ pdf/073rigaut.pdf.

10. J. P. Véran, F. Rigaut, H. Maitre, and D. Rouan, "Estimation of the adaptive optics long-exposure point-spread function using control loop data," J. Opt. Soc. Am. A 14, 3057-3069 (1997).

11. T. Fusco, J.-M. Conan, L. M. Mugnier, V. Michau, and G. Rousset, "Characterization of adaptive optics point spread function for anisoplanatic imaging. Application to stellar field deconvolution," Astron. Astrophys. Suppl. Ser. 142, 149-156 (2000).

12. M. C. Britton, "The anisoplanatic point-spread function in adaptive optics," Publ. Astron. Soc. Pac. 118, 885-900 (2006).

13. R. Flicker, "PSF reconstruction for Keck AO," W. M. Keck Observatory, 65-1120 Mamalahoa Hwy., Kamuela, Hawaii 96743, USA (unpublished). Available online at http://www .oir.caltech.edu/twiki_oir/bin/view/Keck/NGAO/PSF.

14. D. Peter, "Point-spread function reconstruction for the ground layer adaptive optics system ARGOS," Proc. SPIE 7736, 77364R (2010).

15. B. Ellerbroek and F. Rigaut, "Methods for correcting tilt anisoplanatism in laser guide star based multiconjugate adaptive optics," J. Opt. Soc. Am. A 18, 2539-2547 (2001).

16. A. Tokovinin, M. Le Louarn, and M. Sarazin, "Isoplanatism in a multiconjugate adaptive optics system," J. Opt. Soc. Am. A 17, 1819-1827 (2000). 
17. R. Flicker, F. Rigaut, and B. Ellerbroek, "Tilt anisoplanatism in laser guide star based multi conjugate adaptive optics: Reconstruction of the long exposure point spread function from control loop data," Astron. Astrophys. 400, 1199-1207 (2003).

18. L. Gilles and B. L. Ellerbroek, "Constrained matched filtering for extended dynamic range and improved noise rejection for Shack-Hartmann wavefront sensing," Opt. Lett. 33, 1159-1161 (2008).

19. L. Jolissaint, "Synthetic modeling of astronomical closed loop adaptive optics," J. Eur. Opt. Soc. 5, 10055 (2010).

20. C. Correia, J. P. Verant, B. Ellerbroek, L. Gilles, and L. Wang, "Laser-guide star point spread function reconstruction for extremely large telescopes," presented at Adaptive Optics for Extremely Large Telescopes II, Victoria, BC, Canada, 25-30 Sept. 2011, http://ao4elt2.lesia.obspm.fr/sites/ao4elt2/ IMG/pdf/067correia.pdf.

21. L. Gilles and B. L. Ellerbroek, "Real-time turbulence profiling with a pair of laser guide star Shack-Hartmann wavefront sensors for wide-field adaptive optics systems on large to extremely large telescopes," J. Opt. Soc. Am. A 27, A76-A83 (2010).

22. A. Cortes, B. Neichel, A. Guesalaga, J. Osborn, F. Rigaut, and D. Guzman, "Atmospheric turbulence profiling using multiple laser star wavefront sensors," M. Not. R. Astron. Soc. (submitted).

23. L. Wang and B. L. Ellerbroek, "Fast end-to-end multiconjugate $\mathrm{AO}$ simulation using graphical processing units and the MAOS simulation code," presented at Adaptive Optics for Extremely Large Telescopes II, Victoria, BC, Canada,
25-30 Sept. 2011), http://ao4elt2.lesia.obspm.fr/sites/ao4elt2/ IMG/pdf/084wang.pdf.

24. http://lianqiw.github.com/maos.

25. M. Schoeck, S. Els, R. Riddle, W. Skidmore, T. Travouillon, R. Blum, E. Bustos, G. Chanan, S. G. Djorgovski, P. Gillett, B. Gregory, J. Nelson, A. Otarola, J. Seguel, J. Vasquez, A. Walker, D. Walker, and L. Wang, "Thirty Meter Telescope site testing I: overview," Publ. Astron. Soc. Pac. 121, 384-395 (2009).

26. L. Gilles and B. Ellerbroek, "Split atmospheric tomography using laser and natural guide stars," J. Opt. Soc. Am. A 25 , 2427-2435 (2008).

27. L. Wang, B. Ellerbroek, and J. P. Véran, "High fidelity sky coverage analysis via time domain adaptive optics simulations," Appl. Opt. 48, 5076-5087 (2009).

28. L. Wang, B. Ellerbroek, and J. P. Véran, "Full physical optics sky coverage simulation for MCAO systems on ELTs," presented at Adaptive Optics for Extremely Large Telescopes I, Paris, France, 22-26 June 2009, http://dx.doi.org/10.1051/ ao4elt/201003003.

29. J. P. Véran and G. Herriot, "Type II woofer-tweeter control for NFIRAOS on TMT," in Adaptive Optics: Methods, Analysis and Applications, OSA Technical Digest (CD) (Optical Society of America, 2009), paper JTuC2.

30. L. Gilles, L. Wang, and B. Ellerbroek, "Impact of laser launch location on the performance of laser tomography and multi conjugate adaptive optics for extremely large telescopes," Appl. Opt. 49, G114-G119 (2010). 